\title{
Isolated Unilateral Pulmonary Agenesis: A Rare Developmental Disorder
}

\author{
Rudra P Singh ${ }^{1}$, Yashwant Rao ${ }^{1}$, Rupa D Singh ${ }^{1}$, Tanu Midha ${ }^{2}$ \\ ${ }^{1}$ Department of Pediatrics, GSVM Medical College, Kanpur, India, ${ }^{2}$ Department of PSM, GSVM Medical College, Kanpur, India
}

\section{Abstract}

Pulmonary agenesis is a rare developmental defect and is defined as complete absence of lung parenchyma, blood vessels as well as bronchia beyond bifurcation. A 3 day old female neonate presented with tachypnoea and cyanosis. The baby remained on assisted ventilation in NICU for 1 week and then began to deteriorate. CECT was done which showed agenesis of left lung. She expired on 9th day of life.

Keywords: Lung, Pulmonary Agenesis.

Corresponding Author: Dr.Yashwant Kumar Rao L-20, Medical College, Campus, GSVM Medical College, Kanpur, India.

Received: August 2018

Accepted: September 2018

\section{Introduction}

Pulmonary agenesis is a rare developmental defect and is defined as complete absence of lung parenchyma, blood vessels as well as bronchia beyond bifurcation. ${ }^{[1]}$ Left sided agenesis is mostly isolated whereas right lung agenesis is associated with other congenital anomalies. ${ }^{[2]}$

\section{Case Report}

A 3 day old female neonate presented with tachypnoea and cyanosis. She was term baby born by normal vaginal route with birth weight of $2.2 \mathrm{~kg}$. Her parents were non consanguinous. On examination baby showed cyanosis and tachypnoea with a respiratory rate of $72 / \mathrm{min}$ and severe subcostal and intercostal recessions, breath sounds were markedly decreased on the left side. No obvious congenital anomaly was present. Oral intubation was done and baby turned pink on positive pressure ventilation but air entry was absent on left side. Chest radiograph showed hyperinflated right lung with mediastinal shift to left and an opaque left hemithorax. The provisional diagnosis based on chest X-ray and USG chest was collapse of the left lung with mediastinal shift to left. The baby remained on assisted ventilation in NICU for 1 week and then began to deteriorate. CECT was done which showed agenesis of left lung. She expired on 9th day of life.

\section{Discussion \& Conclusion}

Pulmonary agenesis is a rare developmental defect and is defined as complete absence of lung parenchyma, blood vessels as well as bronchia beyond bifurcation. ${ }^{[1]}$ It is thought to be associated recessive trait. About $75 \%$ cases affect left side and are lethal in half of the patients. ${ }^{[2]}$ The survival rate is better with left sided lung agenesis because right lung is larger of the two. Bilateral pulmonary agenesis is incompatible with extrauterine life. Most cases are associated with other congenital anomalies like VACTERAL sequence (Vertebral anomalies, anal atresia, congenital heart defects, tracheo oesophageal fistulas, renal and limb anomalies). ${ }^{[2-5]}$

Unilateral pulmonary agenesis is rare malformation. The embryological origin is likely to be at 4 weeks gestation age and etiology is unknown. Some viral and genetic factors as well as folic acid and vitamin A deficiencies are known to be involved.

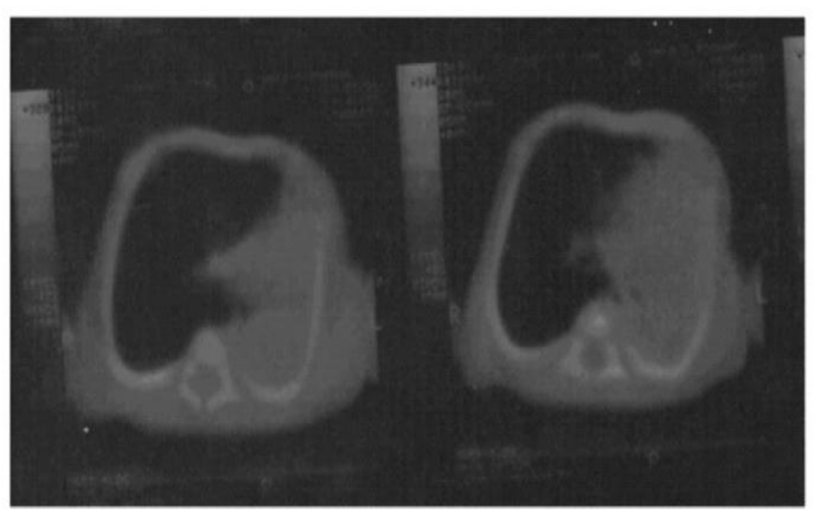

Figure 1: Legend: Contrast enhanced CT of the chest showing left lung agenesis with anterior herniation of right lung.

Incidence is similar in both sexes. ${ }^{[3]}$ Left sided agenesis is mostly isolated whereas right lung agenesis is associated with other congenital anomalies like cardiovascular (Patent ductus arterioses, interruption of aortic arch, total anomalous 
pulmonary venous drainage, scimitar syndrome and hypoplastic left heart syndrome), gastrointestinal (oesophageal atresia and pyloric stenosis), genitourinary (horse shoe kidney), facial (bilateral cleft lip and palate, maxillary and mandibular hypoplasia and bilateral microtia) and skeletal (bilateral radial ray hypoplasia) systems. ${ }^{[3-7]}$ Prognosis is variable and depends on the presenting symptoms and associated congenital malformations that is why right sided agenesis has poorer prognosis. ${ }^{[5]}$ Management is mostly supportive in symptomatic cases with chest physiotherapy and liberal use of antibiotics to treat any pulmonary infections. Death occurs due to respiratory failure. Half of all reported cases die either at birth or within first 5 years of life. Therefore, unilateral lung agenesis should be suspected in symptomatic newborns presenting with respiratory distress refractroy to conventional ventilations as illustrated in this case report.

\section{References}

1. Brombley B, Benacerraf BR. Unilateral lung hypoplasia: report of three cases. J Ultrasound Med 1997;16:599-601.

2. Cunningham ML, Mann N. Pulmonary agenesis: A predictor of ipsilateral malformation. Am J Med Genent 1997;70:391-8.

3. Currarino G, Williams B. Causes of congenital unilateral pulmonary hypoplasia: a study of 33 cases. Pediatr Radiol 1985;15:15-24.

4. Kaya IS, Dilmen U. Agenesis of the lung. Eur Respir J 1989;2:690-2.

5. Kravitz RM. Congenital malformations of the lung. Pediatr Clin North Am 1994:41:453-72.

6. Mardini MK, Nyhan WL. Agenesis of the lung: Report of 4 patients with unusual anomalies. Chest 1985;87:522-7.

7. Osborn J, Masel J, McCredie J. A spectrum of skeletal anomalies associated with pulmonary agenesis: possible neural chest injuries. Pediatr Radiol 1989;19:425-32.

Copyright: (C) the author(s), publisher. Asian Journal of Clinical Pediatrics and Neonatology is an Official Publication of "Society for Health Care \& Research Development". It is an open-access article distributed under the terms of the Creative Commons Attribution NonCommercial License, which permits unrestricted non-commercial use, distribution, and reproduction in any medium, provided the original work is properly cited.

How to cite this article: Singh RP, Rao YK, Singh RD, Midha T. Isolated Unilateral Pulmonary Agenesis: A Rare Developmental Disorder. Asian J. Clin. Pediatr. Neonatol.2018;6(3):7-8.

DOI: dx.doi.org/10.21276/ajcpn.2018.6.3.3

Source of Support: Nil, Conflict of Interest: None declared. 